\title{
15x optical zoom and extreme optical image stabilisation: diffraction limited integral field spectroscopy with the SWIFT spectrograph
}

\author{
Matthias Tecza ${ }^{* a}$, Niranjan Thatte ${ }^{\mathrm{a}}$, Fraser Clarke ${ }^{\mathrm{a}}$, James Lynn ${ }^{\mathrm{a}}$, David Freeman ${ }^{\mathrm{b}}$, Jennifer Roberts $^{\mathrm{c}}$, \\ Richard Dekany ${ }^{\mathrm{d}}$ \\ ${ }^{a}$ University of Oxford, Denys Wilkinson Building, Oxford, OX1 3RH, UK; \\ ${ }^{\mathrm{b}}$ Kidger Optics Associates, 1 Walwyn Mews, Great Malvern, WR14 3DD, UK; \\ ${ }^{\mathrm{c}}$ Jet Propulsion Lab., 4800 Oak Grove Blvd, Pasadena, CA, USA; \\ ${ }^{\mathrm{d}}$ Caltech Optical Observatories, California Institute of Technology, Pasadena, CA, USA
}

\begin{abstract}
When commissioned in November 2008 at the Palomar 200 inch Hale Telescope, the Oxford SWIFT I\&z band integral field spectrograph, fed by the adaptive optics system PALAO, provided a wide $(3 \times)$ range of spatial resolutions: three plate scales of 235 mas, 160 mas, and 80 mas per spaxel over a contiguous field-of-view of $89 \times 44$ pixels. Depending on observing conditions and guide star brightness we can choose a seeing limited scale of 235 mas per spaxel, or 160 mas and 80 mas per spaxel for very bright guide star $\mathrm{AO}$ with substantial increase of enclosed energy.

Over the last two years PALAO was upgraded to PALM-3000: an extreme, high-order adaptive optics system with two deformable mirrors with more than 3000 actuators, promising diffraction limited performance in SWIFT's wavelength range. In order to take advantage of this increased spatial resolution we upgraded SWIFT with new pre-optics allowing us to spatially Nyquist sample the diffraction limited PALM-3000 point spread function with 16 mas resolution, reducing the spaxel scale by another factor of $5 \times$. We designed, manufactured, integrated and tested the new pre-optics in the first half of 2011 and commissioned it in December 2011. Here we present the opto-mechanical design and assembly of the new scale changing optics, as well as laboratory and on-sky commissioning results. In optimal observing conditions we achieve substantial Strehl ratios, delivering the near diffraction limited spatial resolution in the I\&z bands.
\end{abstract}

Keywords: integral field, image slicer, spectrograph, extreme adaptive optics, diffraction limited, fully depleted CCD

\section{INTRODUCTION}

The Oxford SWIFT ${ }^{[1][2]}$ spectrograph is an I\&z band integral field spectrograph (IFS) that was commissioned at the 200inch Hale Telescope on Palomar Mountain in 2008 and is available as a facility instrument to the Palomar community. SWIFT combines the power of adaptive optics (AO) with a high throughput image slicer ${ }^{[3][4]}$ IFS plus a high quantum efficiency detector to give unprecedented sensitivity and resolution at wavelengths between 650 and $1050 \mathrm{~nm}$.

The 200-inch Hale Telescope is an attractive partner for SWIFT because of its strong adaptive optics programme, starting with PALAO ${ }^{[5]}$ (January 1995 - December 1999), continuing with PALMAO (April 2003 - October 2011) with an upgraded the real-time computer and wavefront sensor, adding a Laser Guide Star with PALM-LGS ${ }^{[7]}$ (September 2006 - September 2009), and most recently installing the 3388-actuator deformable mirror in PALM-3000 ${ }^{[8]}$ (from May 2011), enabling extreme AO in the red-visible wavelength range with unprecedented spatial resolution.

\subsection{SWIFT-PALMAO and PALM-LGS}

From the beginning the implementation of SWIFT and the Palomar AO was envisioned to happen in stages. SWIFT was installed at the telescope in October 2008 and the first commissioning run was using PALMAO without the laser guide star. The PALM-LGS mode was commissioned in December 2008. As weather conditions were uncooperative during both runs we only managed to obtain a PALM-LGS data set with the SWIFT acquisition camera. However we demonstrated that PALMAO and PALM-LGS provide $\mathrm{a} \approx 30-50 \%$ decrease of the PSF full width half maximum (FWHM) even under poor conditions (LGS correction reduced the FWHM from 3" natural seeing to 2" PSF FWHM) and a $2 \times$ improvement in enclosed energy in the central spaxel ${ }^{[9]}$.

*matthias.tecza@physics.ox.ac.uk; Tel:+44 (0)1865 273364; Fax: +44 (0)1865 273390

Ground-based and Airborne Instrumentation for Astronomy IV, edited by lan S. McLean, Suzanne K. Ramsay, Hideki Takami, Proc. of SPIE Vol. 8446, 844622 - ( ) 2012 SPIE · CCC code: 0277-786X/12/\$18 - doi: 10.1117/12.925328 


\subsection{SWIFT-PALM3000}

In mid 2010 the PALMAO bench was decommissioned to enable the upgrade to PALM3000. In addition to the 241actuator woofer deformable mirror (DM) of PALMAO, PALM-3000 will use a 3388-actuator tweeter DM to correct wavefront aberrations at scales as fine as $8 \mathrm{~cm}$ at the telescope pupil. Using natural guide stars PALM-3000 will deliver extreme adaptive optics correction at near-infrared wavelengths and diffraction-limited images at visible wavelengths. Already in September 2009 the LGS was indefinitely postponed and as such PALM-3000 prioritises High-contrast observations at both visible and near-IR wavelengths, in particular direct detection and characterisation of extrasolar planets.

In order to exploit the diffraction-limited image quality offered by PALM-3000 and to enable high-contrast observations, we shipped SWIFT back to Oxford and started a programme of instrument refurbishments and upgrades. In the following sections we describe the instrument upgrades, report on the commissioning run and the first on-sky observations.

\section{16 MAS SCALE CHANGING OPTICS}

When first conceived we did foresee that SWIFT should be able to sample a wide range of observing conditions at Palomar, from seeing limited observations with $\approx 1-2$ " resolution over PALM-LGS assisted observations with improved seeing $(\approx 0.5 ")$ to high angular resolution observations with PALM-3000. Correspondingly we designed and built three spaxel scales for SWIFT: 235 mas, 160 mas, and 80 mas. The finest scale of 80 mas was chosen as a compromise between acceptable sensitivity of SWIFT to extended sources, and sampling the PALM-3000 point spread function (PSF) when used with a LGS.

Using bright natural guide stars, however, the PALM-3000 PSF is much narrower and to Nyquist sample the diffraction limited PSF we require a $5 \times$ smaller spaxel size of 16 mas. This means a $5 \times$ larger magnification and therefore a focal length $5 \times$ longer than the 80 mas scale changer. Overall the SWIFT pre-optics covers a $15 \times$ range in magnification from 235 mas to 16 mas per spaxel.

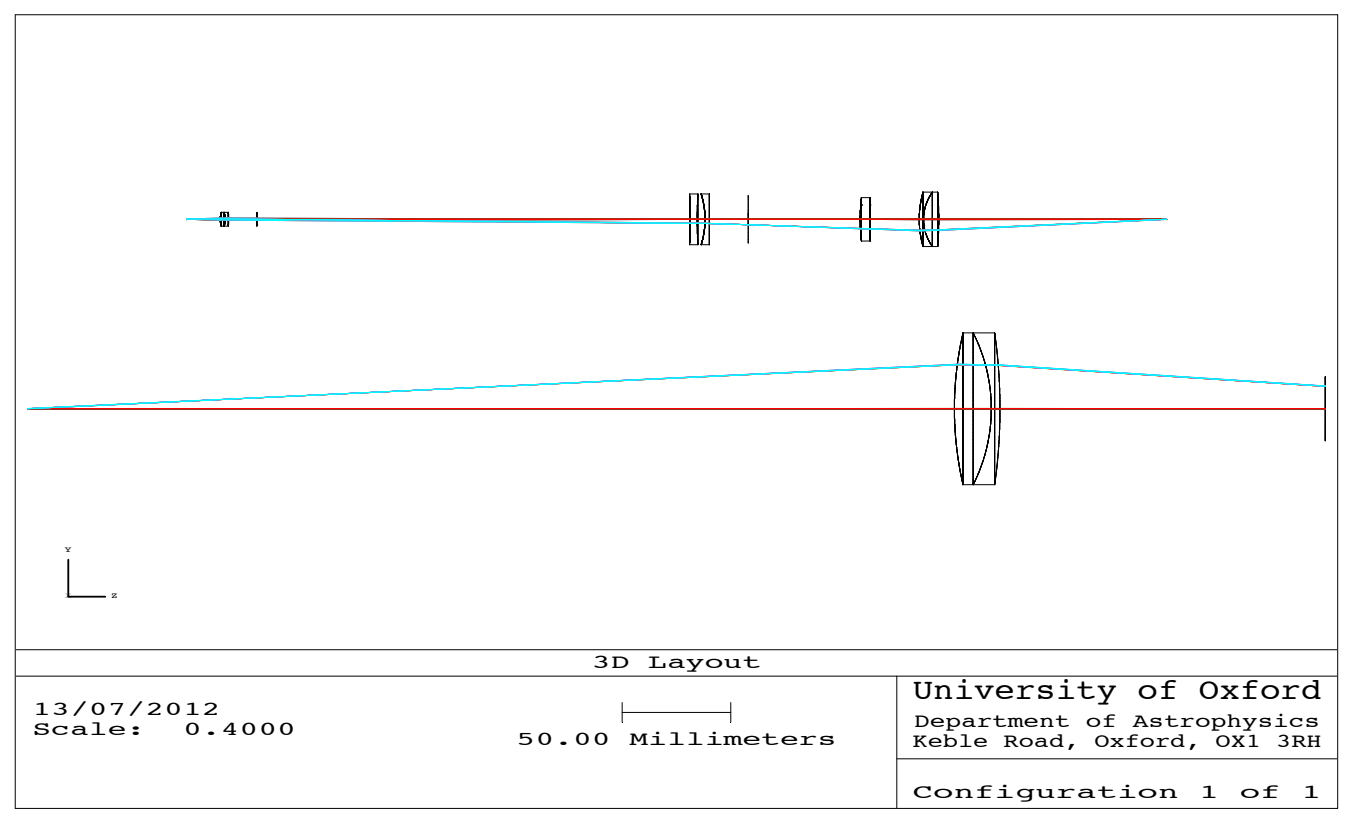

Figure 1: Layout diagrams of the SWIFT 16 mas scale changing optics. The top view shows the optics of the focal reducer and anamorphic beam expander: at the extreme left is the corrected PALM-3000 focal plane, next to it is the achromatic doublet and the pupil baffle, the crossed cylindrical lenses are in the middle, and the re-used SWIFT 160 mas lenses on the right, creating the intermediate pupil image at the extreme right. The bottom view shows the fixed field lens of the SWIFT pre-optics: the intermediate pupil is on the extreme left, and on the right is the image slicer focal plane. 
The SWIFT pre-optics design is divided into two sections: the interchangeable scale changing optics that creates intermediate images of both the PALAO focal plane and the telescope pupil, and the fixed field lens that images the intermediate focal plane onto the image slicer stack and re-images the intermediate telescope pupil to a distance $162 \mathrm{~mm}$ beyond the slicer stack, so as to match the entrance pupil of the image slicer. The scale changing optics comprises two cemented doublets acting as a focal reducer. Both lenses are mounted in tubes that are moved in the beam path by a rotation stage. One requirement for the opto-mechanical design of the new 16 mas scale changing optics was to fit in the exactly the same space envelope of the existing SWIFT scale changing optics. A second requirement was that either catalogue lenses or existing spare SWIFT lenses should be used in the optical design in order to reduce cost and delivery time scales.

The SWIFT pre-optics includes, apart from the scale changer and the field lens, also an anamorphic focal reducer. This is necessary to ensure that we Nyquist sample the spectra while at the same time maximise the field-of-view of SWIFT. While the scale changer provides the correct magnification from the $\mathrm{AO}$ focal plane on to the slicer focal plane along the slices, the anamorphic focal reducer provides a $\times 1 / 2$ anamorphic magnification across the slices. The anamorphic focal reducer consists of two simple cylindrical lenses-one plano-convex and the other plano-concave-forming an anamorphic beam expander. Both cylindrical lenses are mounted in the same tubes as the scale changing optics and are interchanged with it when selecting scales.

Initially we applied the same concept of using a focal reducer plus anamorphic beam expander to the 16 mas scale changing optics, but the resulting design had too short a front focal distance of $\approx 12 \mathrm{~mm}$, the total length of the mounting tube was too long, and the pupil aberrations were unacceptable. However, the disadvantages are avoided if the focal length of the first doublet is increased to $15 \mathrm{~mm}$ and the first cylindrical lens is changed to be toroidal. As off-the-shelf toroidal lenses are almost non-existant two crossed cylindrical lenses mounted close together replace it—one planoconvex and one plano-concave. The resulting optical layout is shown in Figure 1. The first three lenses are off-the-shelf lenses from Thorlabs, the last two lenses are lenses from the SWIFT 160 mas scale changing optics that are re-used.

Image quality of the 16 mas scale changing optics as shown in Figure 2 as a Strehl ratio map. Aberrations are negligible and the design is diffraction limited at all wavelengths over the entire field-of-view. Manufacturing errors have very little effect on the image quality but care must be taken during assembly to achieve the correct first order optical parameters such as back-focal distance, exit pupil location, and magnification. In reality we achieved this easily through the standard mechanical machining tolerances of the Oxford Physics mechanical workshop.

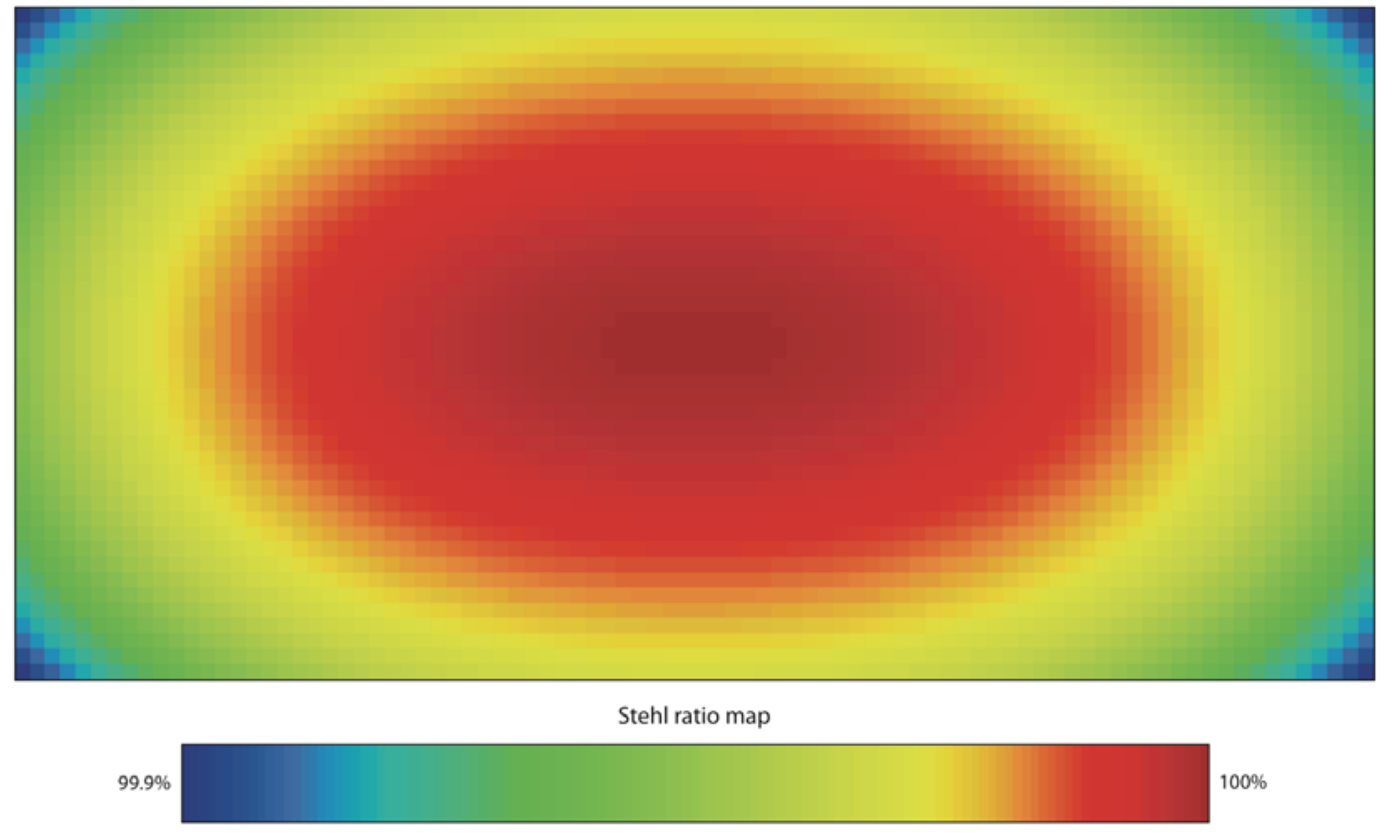

Figure 2: Strehl ratio map of the SWIFT 16 mas spaxel scale at a wavelength of $825 \mathrm{~nm}$ for the $1.4 " \times 0.7$ " field-of-view. Image quality is diffraction limited over the entire field-of-view and at all wavelengths. 

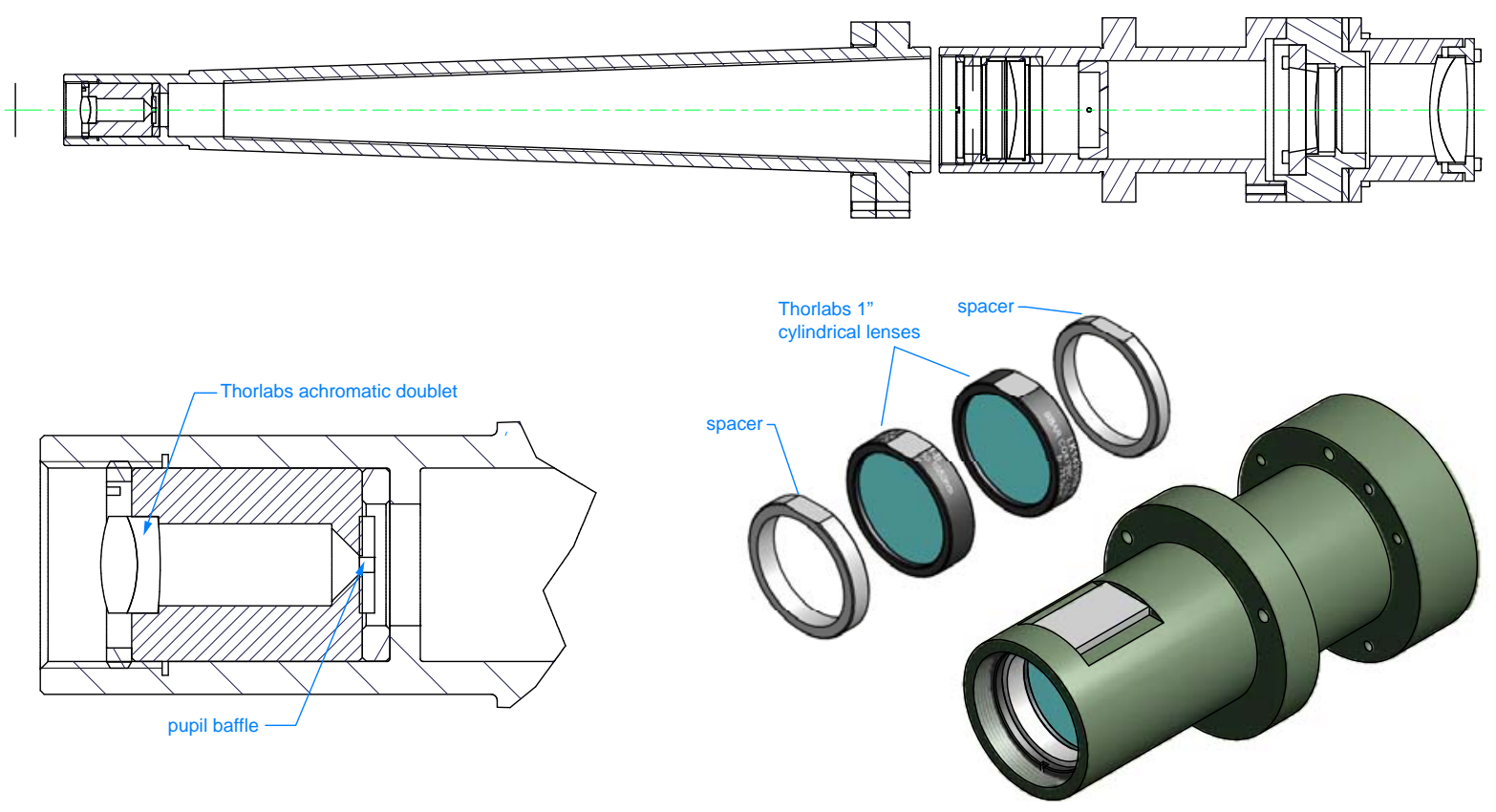

Figure 3: CAD drawings of the SWIFT 16 mas scale changing optics. The top panel shows a cross section view of the entire lens tube. The PALM-3000 corrected focal plane is at the extreme left, next to an achromatic doublet and the pupil baffle mounted in a tapered tube. The tube in the middle holds the crossed cylindrical lenses (see detail view in lower right) and the re-used SWIFT 160 mas lenses in their original mount at the right. The tubes are mounted to a central hub of the rotation stage that also holds the 235 mas and 80 mas scale changing optics (see also Figure 4). The lower left detailed view shows how the achromatic doublet and the pupil baffle are mounted. At the lower right we show an assembly view of the lens tube holding the crossed cylindrical lenses. The machined flats on the mounted lenses indicate the cylinder axis, which were determined using a coordinate measurement machine.

As mentioned above, all SWIFT scale changing optics are assembled in lens tubes that are in turn mounted in a revolver arrangement to the central hub of a rotation stage (see Figure 4). CAD drawing of the 16 mas scale changing lens tube is shown in Figure 3. The tube is split into three sections: a long tapered tube holding the first achromatic doublet and the pupil baffle, a second tube holding the crossed cylindrical lenses, and the third tube holding the re-used lenses from the 160 mas scale changing optics. The interface between the second and the third tube is designed such as to re-use the 160 mas opto-mechanics unmodified.

The relative positioning tolerance between the achromatic doublet and the pupil baffle is rather stringent as otherwise the telescope pupil image will be vignette by the pupil baffle. We therefore integrate the lens mount and pupil baffle in a precision-machined tube that in turn is inserted in the tapered lens tube (see Figure 3). To further minimize the impact of manufacturing errors we measured the outer diameter of the Thorlabs doublet and machined the integrated lens mountpupil baffle accordingly.

As the cylindrical lenses from Thorlabs do not have their axes marked, we had to devise a method to determine their orientation and machine alignment features on their mounts to be able to correctly assembly them in the scale changer tube. For that purpose we mounted the lenses in a holder and used the coordinate measuring machine (CMM) of the Oxford Physics mechanical workshop to determine the cylinder axis relative to the reference surfaces on the lens holder. The same reference surfaces we then used on a CNC mill to accurately machine flat alignment surfaces to the lens mounts, as can be seen in Figure 3.

The assembled and integrated 16 mas scale changing optics is shown in Figure 4. The CAD rendering on the left shows fold mirror FM3 (top left corner) reflecting the light from PALM-3000 towards the 16 mas scale changing tube. Between FM3 and the narrow end of the tapered 16 mas tube is the focal plane mask wheel that holds aperture masks used for calibration purposes. In the lower right corner is the shutter and a fold mirror, folding the light path upwards towards another fold mirror that directs the light towards the field lens (in the top right corner) and into the image slicer (not shown). The photograph on the right shows the same view of the SWIFT pre-optics as installed at the telescope. 

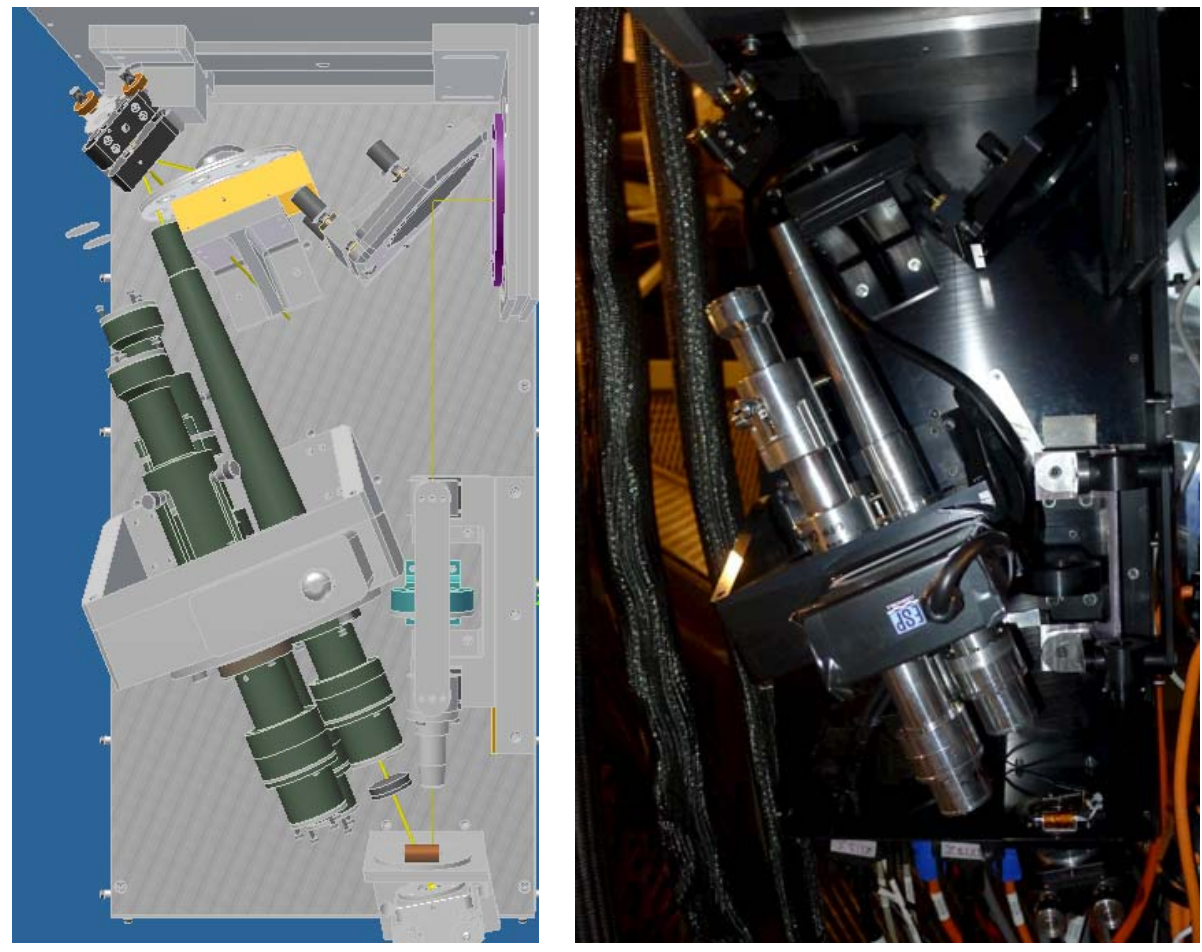

Figure 4: CAD rendering and photograph of the SWIFT pre-optics. In the CAD rendering we show the three SWIFT scale changers for 235 mas, 80 mas, and 16 mas, mounted in a revolver arrangement on a Newport rotation stage. In the middle of photograph on the right is the 16 mas lens tube next to the 80 mas scale changing optics.

\section{3. "BLADE CORONAGRAPH"}

SWIFT was originally designed as a very sensitive IFS for extragalactic astronomy ${ }^{[1]}$, for example measuring the Rotation curves and dynamical masses out to $z=1.7$, studying the Stellar dynamics in the innermost regions of nearby galaxies, or the Kinematics and Dynamics of Ly- $\alpha$ emitters at $5<z<7$. However, as PALM-3000 does not have a LGS, SWIFT's main scientific goal shifted towards high-contrast observations of bright targets. Especially the search for and characterisation of low-mass companions requires extreme $\mathrm{AO}$ correction, which is only possible with bright guide stars. One problem with these bright guide stars is that in order to not saturate the SWIFT CCD detector the exposure times are very short, typically less than a few seconds. Not only is the signal-to-noise ratio of these short exposures dominated by the CCD read-noise, the observing efficiency is also poor because of the long CCD read-out time of $\approx 90$ s. Ideally we would like to occult the bright star at the entrance to SWIFT and use a coronagraph like other high contrast instruments to, however, this cannot be retrofitted to the SWIFT pre-optics. Instead we added an occulting blade behind the image slicer, blocking the central slices of the SWIFT FoV. Figure 5 shows four SWIFT flat field illuminations with and without the occulting blade in the SWIFT FoV. The blade mechanism is actuated by a Hitec HS-325 HB Servo, controlled by a PhidgetAdvancedServo 1-Motor servo controller attached to a USB port of the SWIFT instrument control computer. Both the servo controller and the Servo are USB powered greatly simplifying their installation.

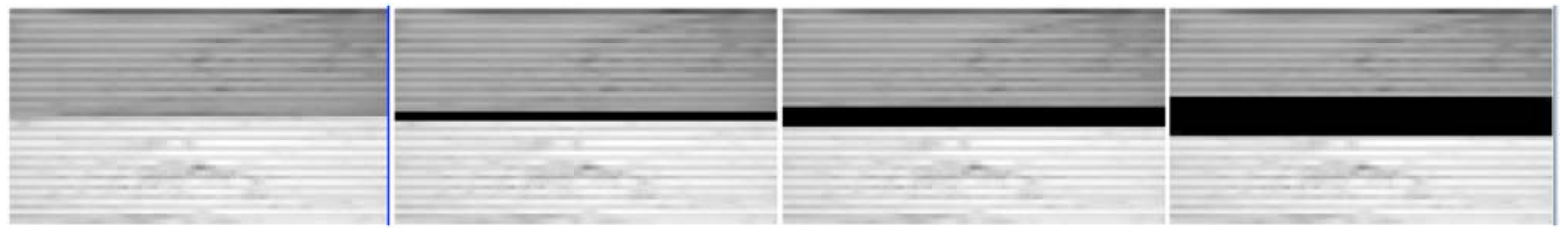

Figure 5: Reconstructed SWIFT flat-field illuminations illustrating the blade coronagraph. From left-to-right we show an un-occulted SWIFT flat field, the blade "coronagraph" covering two, four and eight slicer, respectively. 


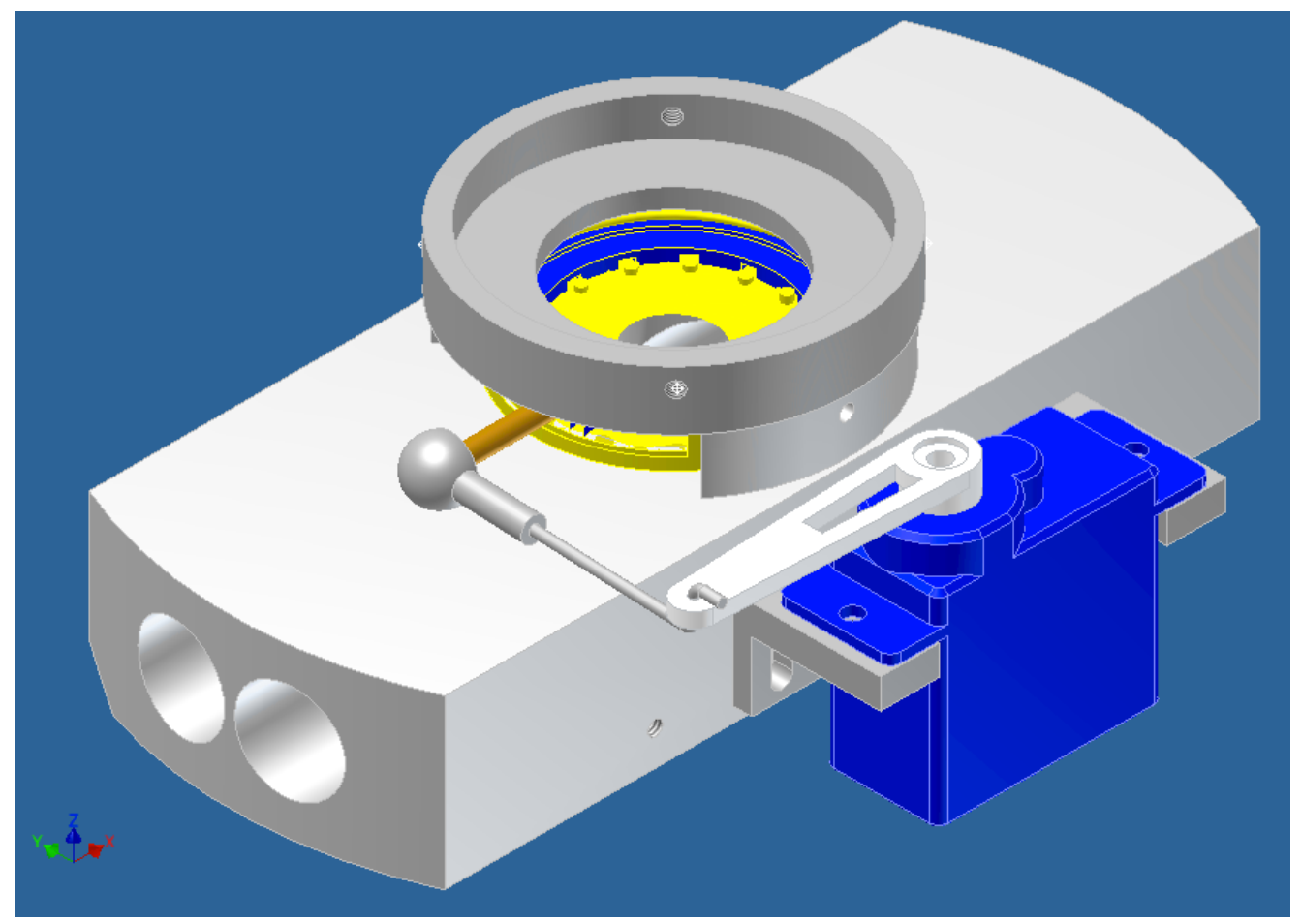

Figure 6: CAD rendering of the SWIFT arc-lamp holder with the additional IRIS aperture (yellow/blue) and Servo (blue).

The IRIS aperture is sandwiched between the original arc-lamp holder (light grey, with two holes for the Argon and Neon lamp, respectively) and an adapter ring (dark grey). The adjustable IRIS is actuated by a Servo connected by a ball joint link.

\section{CALIBRATION UNIT}

Increasing the zoom range of the SWIFT pre-optics by a factor of $5 \times$ has a significant impact on its calibration unit. In the original design of SWIFT we had a factor $3 \times$ zoom range ( 235 mas to 80 mas), and a hence a factor of $\approx 10 \times$ in flux from the calibration unit. This is especially the case for the Argon and Neon arc-lamps-used for wavelength calibration-as their intensity cannot be controlled. To compensate for this range of source fluxes we simply increased the exposure times from 1 second in the 235 mas scale to 10 seconds in the 80 mas scale. This was acceptable as the CCD readout time of $\approx 90$ seconds is much larger.

However, SWIFT now covers a $15 \times$ range of spaxel size, which means a factor $225 \times$ range in flux per pixel for the same calibrations source, which makes the exposure time of the arc-lamps prohibitively long. In order to control the flux of the arc-lamps we therefore added and adjustable IRIS aperture stop (see Figure 6) to the SWIFT calibrations unit. The IRIS stop is actuated by a Hitec HS-55 Servo, and controlled by a PhidgetAdvancedServo 1-Motor servo controller attached to a USB port of the SWIFT instrument control computer. Both the servo controller and the Servo are USB powered greatly simplifying their installation.

\section{PREMIMINARY RESULTS}

After we successfully integrated and tested the 16 mas scale changing optics in Oxford in September 2011, we shipped the upgraded instrument to Palomar. In October 2011 we integrated SWIFT and PALM-3000 in the Palomar AO-lab, aligned the pupil and focus of both instruments and determined the relative field centres of all three SWIFT scales.

In early December 2011 we re-commissioned SWIFT at PALM-3000 on the 200 inch Hale Telescope on Palomar. Unfortunately bad weather and seeing of $\approx 2 "-4$ " stopped us from using the 16 mas scale on sky, however, PALM-3000 was working and we were able to use the AO calibration point source to test the image quality of SWIFT/PALM-3000. From these tests we found that non-common path (NCP) errors between PALM3000 and SWIFT limit the internal image quality to a FWHM $\approx 0.1 "$. This can be improved by applying a static pattern to the deformable mirror of PALM-3000. 
Our next opportunity to use SWIFT/PALM-3000 was during a four-night observing run in July 2012. This time the weather was cooperative and we had four clear nights with seeing in the range from 1.0"-1.5". In addition to the good intrinsic atmospheric image quality we minimised the NCP wavefront error improve the SWIFT PSF. Using the PALM3000 calibration point-source, we manually varied the wavefront error by applying different amounts of Zernike aberrations to the deformable mirror, visually compared the resulting images and selected the best PSF. We did this in turn for the ten first Zernike aberrations. The final PSF at a wavelength of $825 \mathrm{~nm}$ is shown in Figure 7. The FWHM of the optimised PSF is 40 mas, only a factor $1.2 \times$ broader than the perfect Airy pattern.

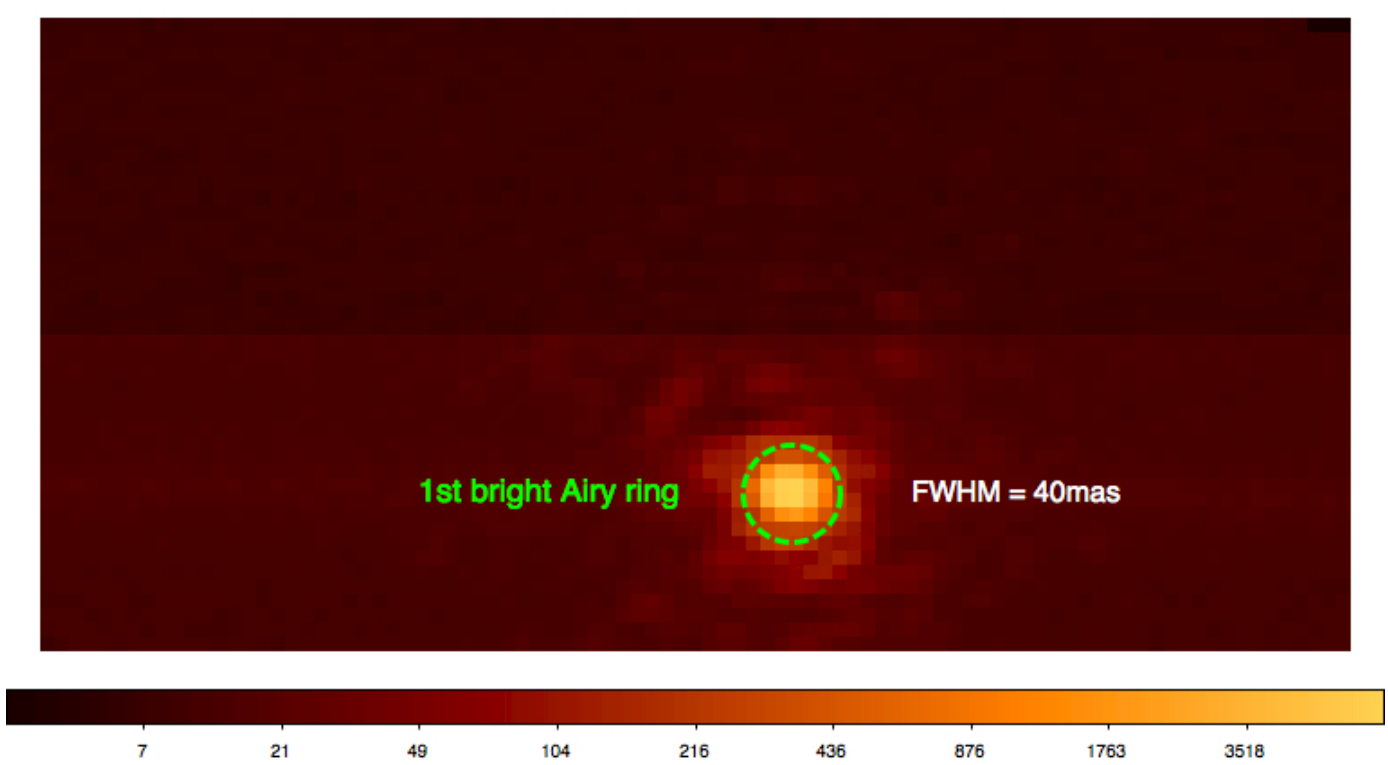

Figure 7: SWIFT/PALM-3000 PSF at $\lambda \approx 800 \mathrm{~nm}$, shown in a logarithmic scaling. The green, dashed circle denotes the position of the first bright Airy ring, the central core of the PSF has a FWHM=40 mas, the FWHM of a perfect Airy pattern in comparison is 33 mas.

After we optimised the SWIFT PSF we tested the instrument resolution during the same night by observing a known ${ }^{[10]}$ close binary-SAO 85767. This binary has a separation of 0.116 " and a contrast of $\Delta \mathrm{m} \approx 3$ mag. The resulting narrow band image (at $\lambda \approx 800 \mathrm{~nm}$ ) is shown in Figure 8 . SWIFT clearly resolves this binary, and achieves a resolution of 50 mas, as measured by the FWHM of the primary star, showing that the Palm-3000 extreme adaptive optics can deliver extremely sharp images even in the red-visible wavelength range, but also that the control and correction of the NCP aberrations is critical for high-contrast observations with SWIFT. This opens up exciting opportunities for highresolution integral-field astronomy of bright targets, especially extended solar system objects.

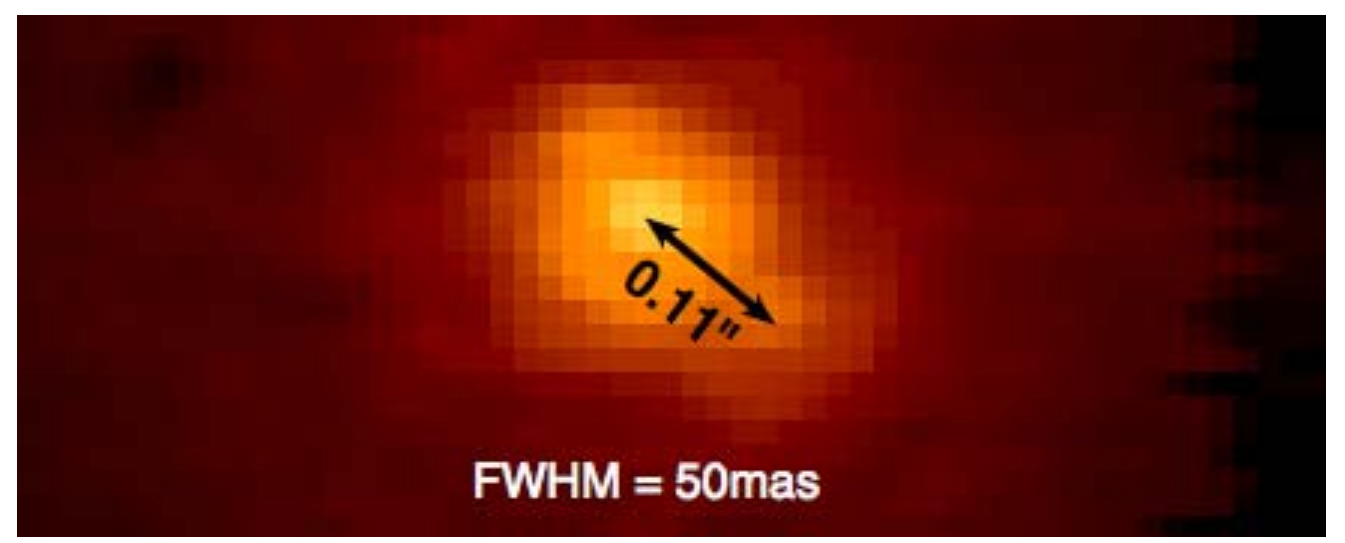

Figure 8: Narrow band image at $\approx 800 \mathrm{~nm}$ of the close binary star SAO 85767 . The binary system with a separation of $0.116 "$ and contrast of $\approx 3$ mag is clearly resolved. The FWHM of the primary star is only 50 mas. 


\section{ACKNOWLEDGEMENTS}

The SWIFT team would like to express their sincere gratitude to the Palomar Observatory staff and the PALM-3000 team, in particular Jean Mueller, Kevin Rykoski, Jenny Roberts, Rick Burruss and Rich Dekaney for their generous help during the installation, commissioning and subsequent operations of the SWIFT/PALM-3000.

Based on observations obtained at the Hale Telescope, Palomar Observatory, as part of a collaborative agreement between the California Institute of Technology, its divisions Caltech Optical Observatories and the Jet Propulsion Laboratory (operated for NASA), and Cornell University.

\section{REFERENCES}

[1] Tecza, M. et al., "SWIFT: An adaptive optics assisted I/z band integral field spectrograph," New Astronomy Reviews 49, 647-654 (2006)

[2] Thatte, N. et al., "The Oxford SWIFT integral field spectrograph," Proc. SPIE 6269, 62693L (2006)

[3] Tecza, M. et al., "SWIFT image slicer: large format compact low scatter image slicing," Proc. SPIE 6273, 62732L (2006)

[4] Tecza, M. et al., "SWIFT de-magnifying image slicer: diffraction limited image slicing at optical wavelengths," Proc. SPIE 7018, 70182O-70182O-9 (2008)

[5] Troy, M. et al., "Palomar adaptive optics project: status and performance," Proc. SPIE 4007, 31-40 (2000)

[6] Bouchez, A.H. et al., "Status of the PALM-3000 high-order adaptive optics system," Proc. SPIE 7736, 77361Q77361Q-7 (2010)

[7] Roberts, J.E. et al., "Facilitizing the Palomar AO laser guide star system," Proc. SPIE 7015, 70152S-70152S-10 (2008)

[8] Bouchez, A.H. et al, "Status of the PALM-3000 high-order adaptive optics system," Proc. SPIE 7736, 77361Q77361Q-7 (2010)

[9] Thatte, N. et al., "The Oxford SWIFT Spectrograph: first commissioning and on-sky results," Proc. SPIE 7735, 77357Y-77357Y-14 (2010)

[10] Horch, E.P. et al., "CCD Speckle Observations of Binary Stars with the WIYN Telescope. VI. Measures During 2007-2008," The Astronomical Journal 139, 205-215 (2010) 